\title{
Three Dimensional Subsurface Elemental Identification of Minerals using Confocal Micro X-ray Fluorescence and Micro X-ray Computed Tomography
}

Nikolaus L. Cordes ${ }^{1, a}$, Srivatsan Seshadri ${ }^{2, a}$, George J. Havrilla ${ }^{3}$, Xiaoli Yuan ${ }^{4}$, Michael Feser $^{2}$ and Brian M. Patterson ${ }^{1}$

${ }^{1}$ Polymers and Coatings Group, Material Science and Technology Division, Los Alamos National Laboratory, Los Alamos, NM 87545, USA

${ }^{2}$ Carl Zeiss X-Ray Microscopy, Inc., Pleasanton, CA 94588, USA

${ }^{3}$ Chemical Diagnostics and Engineering, Chemistry Division, Los Alamos National Laboratory, Los Alamos, NM 87545, USA

${ }^{4}$ Julius Kruttschnitt Mineral Research Centre, University of Queensland, Indooroopilly, Brisbane, QLD 4068, Australia

${ }^{\mathrm{a} C}$ Corresponding authors.

Tel: +1 925701 3615. Email: srivatsan.seshadri@zeiss.com (Srivatsan Seshadri)

Tel: +1 505695 8049. Email: ncordes@lanl.gov (Nikolaus L. Cordes)

Keywords: confocal micro X-ray fluorescence; micro X-ray computed tomography; subsurface particle analysis

\begin{abstract}
Current non-destructive elemental characterization methods, such as scanning electron microscopy-based energy dispersive spectroscopy (SEM-EDS) and micro X-ray fluorescence spectroscopy (MXRF), are limited to either elemental identification at the surface (SEM-EDS) or suffer from an inability to discriminate between surface or depth information (MXRF). Thus, a non-destructive elemental characterization of individual embedded particles beneath the surface is impossible with either of these techniques. This limitation can be overcome by using laboratory-based 3D confocal micro X-ray fluorescence spectroscopy (confocal MXRF). This technique utilizes focusing optics on the X-ray source and detector which allows for spatial discrimination in all three dimensions. However, the voxel-by-voxel serial acquisition of a 3D
\end{abstract}


elemental scan can be very time-intensive ( 1 to 4 weeks) if it is necessary to locate individual embedded particles of interest. As an example, if each point takes a $5 \mathrm{~s}$ measurement time, a small volume of $50 \times 50 \times 50$ pixels leads to an acquisition time of approximately 174 hours, not including sample stage movement time. Initially screening the samples for particles of interest using micro X-ray computed tomography (micro-CT) can significantly reduce the time required to spatially locate these particles. Once located, these individual particles can be elementally characterized with confocal MXRF. Herein, we report the elemental identification of high atomic number surface and subsurface particles embedded in a mineralogical matrix by coupling microCT and confocal MXRF. Synergistically, these two X-ray based techniques first rapidly locate and then elementally identify individual subsurface particles.

\section{Introduction}

Elemental identification of coatings, layers, particles, or contaminants is of great interest within many scientific disciplines, including chemistry, materials science, archaeology, paleontology and mineralogy. Several characterization techniques are available for qualitative elemental analysis of embedded particles. The choice of the appropriate technique for particle analysis depends on several factors such as analysis time, sample preservation, limits of detection, and spatial location of the region of interest within the sample (i.e., surface or subsurface). Destructive analytical methods may involve the chemical dissolution[1] or laser ablation[2] of a sample followed by analytical characterization techniques such as inductively coupled plasma mass spectrometry.[3] Often, non-destructive microscopy-based methods, such as scanning electron microscopy-based energy dispersive spectroscopy (SEM-EDS), are used to identify the elements comprising a sample. However, this method is restricted to the near surface (typically 1 to $5 \mu \mathrm{m}$, depending on sample composition) due to the short penetration depth, 
electron-matter interaction of exciting electrons, and attenuation of emitted x-ray photons by the sample material. Using this method to probe subsurface regions of interest typically requires destructive methods, such a focused ion beam (FIB) milling to access regions below the surface. Characterization techniques based on X-ray excitation, such as micro X-ray fluorescence spectroscopy (MXRF), are capable of probing subsurface regions. In traditional MXRF, a polycapillary focusing lens is commonly employed to focus an X-ray tube source, limiting the excitation spot diameter on the sample to the range of $\sim 20 \mu \mathrm{m}$ to $\sim 100 \mu \mathrm{m}$. However, there is no discrimination regarding the origin of the $\mathrm{x}$-ray photons detected, making it impossible to differentiate between surface and subsurface $\mathrm{x}$-ray fluorescent photons with this characterization technique.

The use of SEM operating in back-scattered electron (BSE) mode is prevalent within the mining community as a method for the automated analysis of individual mineralogical particles within standardized mineralogical molds. Termed mineral liberation analysis (MLA), this method uses an SEM equipped with a BSE detector and EDS which allows for the differentiation of unique mineral phases within a sample. MLA is a powerful tool for quick identification of mineral phases within small particles; however, one disadvantage of this technique is the inability to probe beneath the surface of the mineralogical molds in a non-destructive manner. While a comprehensive survey of MLA is beyond the scope of this article, an informative review by Fandrich et al. can be found in reference [4].

Both the depth discrimination limitation of MXRF and surface restriction limitation of SEM-EDS can be overcome by using confocal MXRF (also referred to in the literature as 3D MXRF)[5-10], which is achieved by placing one polycapillary focusing lens on the X-ray source and another on the detector (Fig. 1). With these two optics arranged in a confocal geometry 
(typically from $30^{\circ}$ to $45^{\circ}$ from the surface normal), it is then possible to spatially discriminate X-ray fluorescence emission in all three spatial directions. This is achieved by moving the region of interest of a sample into the confocal excitation/detection volume using a set of lateral XY stages and a vertical $\mathrm{Z}$ stage. The confocal geometry creates a spatially constricted ellipsoidal[11] or spherical[12] X-ray excitation/detection volume, which allows for the nondestructive elemental analysis of surface and subsurface regions of interest, such as stratified layers[8], paint layers[13], embedded metal-doped polymer layers[14, 15], and lithium-ion battery cathodes.[16] However, one drawback of confocal MXRF is the time required to obtain full 3D elemental maps, which can vary from days to weeks, depending on the energy of the Xray source, sample composition, sample size, desired horizontal and vertical resolutions, and dwell time needed to obtain an appropriate signal-to-noise ratio. The analysis time of confocal MXRF can be significantly reduced by screening for particles of interest using micro-CT, as described below.

Micro X-ray computed tomography (micro-CT) is a non-destructive 3D imaging technique in which a series of X-ray projection radiographs are collected as the sample is rotated by at least $180^{\circ}$. These images are then mathematically reconstructed to yield a stack of $2 \mathrm{D}$ images, which can be rendered in 3D using visualization software. Such 3D images obtained with micro-CT consist of a cubic array of voxels with average attenuation values represented by grayscale values. The differences in grayscale values correspond to differences in attenuation and are representative of the electron density of the material that caused this attenuation. A histogram of these grayscale values allows one to analyze and determine the various materials and their phases present in the image provided these values are suitably calibrated. The imaging contrast provided by the grayscale values are often sufficient for many applications in scientific 
disciplines, such as geoscience[17], for the imaging of embedded features; material science, for the imaging of void structures within polymer foams[18, 19]; in paleontology, for the imaging of fossilized embryos[20]; and in mineral processing, for mineral exposure[21] and liberation[22, 23] analysis. A disadvantage of micro-CT is that it does not yield specific elemental information (which can be obtained from confocal MXRF); however, high absorption particles embedded in a low absorption matrix can easily be detected based on regions of concentrated high grayscale values.

Herein, we report the qualitative elemental analysis of particles of interest composed of high atomic number elements within packed mining tailings embedded in epoxy using laboratory-based confocal MXRF and laboratory-based micro-CT in an integrated manner. To date, this is the only known study integrating these two laboratory-based techniques for the application of mineral analysis. Full 3D data sets obtained by micro-CT provide a global inspection of the sample by identifying particles of interest based on X-ray attenuation. While this is not elemental information, it can serve as an indication of potential particles of interest given that the particle is embedded in mostly a low atomic number element matrix (e.g., silicate minerals). Fiducials are then placed on the surface of the sample to indicate the location of surface particles and the two dimensional location of subsurface particles. The sample was then transferred to the confocal MXRF instrument. The elemental signal of the fiducial markers (W and $\mathrm{Au}$ ) allowed for rapid location of the particles of interest and their elemental identification.

\section{Experimental}

\subsection{Procedure for Analysis}


The analysis procedure is as follows: First, the mining tailings were embedded in the epoxy resin (described in Section 2.2) and micro-CT imaging was performed (described in Section 2.3). Micro-CT imaging was then repeated for the other epoxy-embedded mining tailing samples. From the micro-CT data, high Z particles of interest were identified by the particles' grayscale values and their locations were noted. Then, all seven epoxy-embedded mining tailings samples were embedded into a larger epoxy puck. This puck was then polished such that at least one $\mathrm{Pt}$ or $\mathrm{Pb}$ particle was present at the surface of the tailing specimen of interest. The surface of the puck was then analyzed by MLA (described in Section 2.4), providing both spatial location (SEM) and elemental identification (EDS) of the particles. The elemental identification via SEM-EDS was used as a control for the confocal MXRF analysis. Gold-plated tungsten wires were placed near the surface particle of interest and near the lateral position of the subsurface particle to aid in the alignment of the sample in the confocal MXRF microscope and to minimize the "scouting" time required to locate the particles with confocal MXRF.. The epoxy puck was then transferred to the confocal MXRF microscope and aligned using the gold-plated tungsten wires. The sample was then systematically scanned for the surface high $\mathrm{Z}$ particle of interest. The confocal MXRF fluorescence signal from the surface particle was then compared to the MLA results. Once it was confirmed that the confocal MXRF and MLA results matched, the confocal MXRF procedure was repeated for the subsurface particle (described in Section 2.5).

\subsection{Sample Preparation}

A cylindrical sample of $\sim 1.67 \mathrm{~mm}$ diameter and $\sim 4 \mathrm{~mm}$ in height, consisting of mineral particles (ranging from $10 \mu \mathrm{m}$ to $40 \mu \mathrm{m}$ in diameter) embedded in epoxy resin, was prepared from mining tailings (Fig. 2). Some of these mining tailing particles contained either Pt- or Pbbearing minerals. The sample was first imaged using micro-CT (described in the next section) to 
identify the spatial location of high density particles of interest. Later, the sample was embedded into an epoxy puck of $30 \mathrm{~mm}$ diameter and $~ 17 \mathrm{~mm}$ height along with six other epoxy-embedded mining tailing samples. The surface of the puck was then polished until at least one $\mathrm{Pt}$ or $\mathrm{Pb}$ particle was present at the surface.

\subsection{Micro X-ray Computed Tomography}

Before embedding into the epoxy puck, the sample was imaged on an Xradia (now Carl Zeiss X-ray Microscopy, Inc., Pleasanton, CA) 3D X-Ray microscope, the MicroXCT-200 system equipped with a 10X objective lens, at an accelerating voltage of $90 \mathrm{kVp}$ and operating power of $8 \mathrm{~W}$. The sample was rotated $360^{\circ}$ using $0.5^{\circ}$ steps per projection and $10 \mathrm{~s}$ exposure per projection, resulting in a total of 721 projections and data acquisition time of $2 \mathrm{~h}$. The resulting tomogram exhibited an isotropic voxel size of $1.9 \mu \mathrm{m}$. High density particles of interest were located by identifying regions of high grayscale value within the tomograms. This was accomplished by arbitrarily choosing a high grayscale threshold value and segmenting the tomogram.

The X-ray tomographic data was visualized and analyzed using Avizo Fire Edition version 8.0 software (FEI Visualization Sciences Group, Burlington, MA). Equivalent diameter and volume measurements of particles were calculated by segmenting the data set for the grayscale values corresponding to the particles of interest, and then applying a label field analysis to the binary segmented image data set. Volume renderings of the segmented images were generated using Avizo's Volume Rendering module.

\subsection{Mineral Liberation Analysis}


The mineral sample embedded in the polished epoxy puck was measured using MLA (details described elsewhere[4, 24] ) at Julius Kruttschnitt Mineral Research Centre (JKMRC, http://www.jkmrc.uq.edu.au/) to obtain an X-ray identification of the particles at the surface. First, BSE micrographs were acquired of the high density particles on the surface followed by EDS X-ray spectra (data not shown). The elemental composition of one surface particle was determined by MLA to be tetraferroplatinum. To ensure rapid location of particles of interest with the confocal MXRF microscope, gold-plated tungsten wires, $25 \mu \mathrm{m}$ in diameter, were used as fiducial markers and placed on the surface of the sample. SEM micrographs of samples containing these markers are shown in Figure 3. The sample was then probed with the confocal MXRF microscope at Los Alamos National Laboratory (Los Alamos, NM), to identify the elemental composition of the chosen surface particle and compare with the results obtained with MLA. In addition, subsurface particles were also chosen for elemental identification and studied with confocal MXRF.

\subsection{Confocal Micro X-ray Fluorescence Spectroscopy}

Confocal micro X-ray fluorescence spectroscopy was performed using a custom confocal MXRF microscope, which was developed jointly by Los Alamos National Laboratory and X-ray Optical Systems (XOS, East Greenbush, NY). The microscope uses a pair of XOS monolithic fused-silica polycapillary optics which are placed in front of both the XOS X-beam Ag tube Xray source and Amptek Model XR-100CR Si-pin diode detector (Amptek Inc., Bedford, MA). The working distance of the microscope is approximately $10 \mathrm{~mm}$. Three $850 \mathrm{G}$ Newport linear stages allow for movement of the sample in the lateral $\mathrm{X}$ and $\mathrm{Y}$ planes as well as the vertical $\mathrm{Z}$ plane and are controlled by a Newport motion controller (Newport Corporation, Irvine, CA). The source, detector, and motion controller are interfaced with a Dell Optiplex computer running a 
custom Labview (National Instruments Corporation, Austin, TX) program used for instrument control and collection of the detector output. The dimensions of the confocal excitation/detection volume was determined to be $30 \mu \mathrm{m}$ x $30 \mu \mathrm{m}$ x $50 \mu \mathrm{m}\left(\sim 188,500 \mu \mathrm{m}^{3}\right.$, calculated as a prolate spheroid) from scans using a $10 \mu \mathrm{m}$-thick Ta foil using the Ta L $\alpha$ X-ray emission line (8.14 $\mathrm{keV}$ ); however, these dimensions will vary slightly depending on the X-ray fluorescent energy.[15] For the current study, confocal MXRF scans were performed using an accelerating voltage of $45 \mathrm{kVp}$ and total power of $20.25 \mathrm{~W}$. Data collection times, as well as lateral and vertical step sizes, for each analysis are stated in the Results section. Confocal MXRF 2D elemental maps were rendered using OriginPro 8.6.0 (OriginLab Corporation, Northampton, MA). Generally, confocal MXRF scans were performed by initially searching for the W L $\alpha$ fluorescent signal of the indicator wire in the lateral $\mathrm{X}$ and $\mathrm{Y}$ directions. Once the indicator wire was located, a 2D elemental map of the indicator wire was obtained. From the spatial location of particles of interest obtained from micro-CT data, successive 2D elemental maps were then obtained in the location of interest as a function of depth until the particle of interest was located. Spectra were then acquired with the confocal MXRF excitation/detection volume centered over the particle of interest to confirm the elemental identification of the particle. The X-ray fluorescent energy regions of interest (ROI) and their energy ranges used in obtaining 2D elemental maps are listed in Table 1.

\section{Results and Discussion}

\subsection{Spatial Location of Particles of Interest}

Figure 3A shows a SEM micrograph of the surface of a portion of a mineral sample, $\sim 1.67 \mathrm{~mm}$ in diameter, embedded in an epoxy puck. A gold-plated tungsten wire can be seen, 
indicating the location of the particle on the surface of the mineral sample, designated as Particle A. This particle was identified by MLA as a tetraferroplatinum particle, as described earlier, and is located approximately $100 \mu \mathrm{m}$ from the tip of the gold-plated tungsten indicator wire. The lateral position of the subsurface particle of interest, designated as Particle B, is indicated by the gold-plated tungsten wire depicted in Figure 3B.

Figure 4 is a set of micro-CT reconstructed slices arranged to highlight the surface location of Particle A and subsurface location of Particle B. Particle A can easily be seen on the surface slice. Particle B, which is located $\sim 300 \mu \mathrm{m}$ below the surface, can be seen on the depth slice. Particle B was identified as a particle of interest by its micro-CT grayscale value. This particle was also chosen for further characterization based on its depth beneath the surface; this depth was estimated to be within the limit of the confocal MXRF microscope detection depth for a high $\mathrm{Z}$ particle embedded within a mineral sample matrix. From the micro-CT data set, the equivalent diameter (defined by Equation 1) and volume ( $V$ ) of Particle A was calculated to be $19.1 \mu \mathrm{m}$ and $3664 \mu \mathrm{m}^{3}$, respectively. The equivalent diameter and volume of Particle B was determined to be $19.5 \mu \mathrm{m}$ and $3875 \mu \mathrm{m}^{3}$, respectively.

Equivalent Diameter $=2\left(\sqrt[3]{\frac{3 V}{4 \pi}}\right)$ Equation 1

\subsection{Detection of Particles of Interest using Confocal MXRF}

\subsubsection{Surface - Particle A}


Figures 5A-5I presents a series of 2D elemental maps of W L $\alpha$ energy ROI (Figs. 5A, 5B, and 5C), Pt L $\alpha$ energy ROI (Figs. 5D, 5E, and 5F), and Fe K $\alpha$ energy ROI (Figs. 5G, 5H, and 5I), recorded near the tip of the gold-plated tungsten wire indicating the spatial location of Particle A. These elemental maps are $250 \mu \mathrm{m} \times 400 \mu \mathrm{m}$, and were recorded using $3 \mu \mathrm{m}$ step sizes in the $\mathrm{X}$ and $\mathrm{Y}$ directions, with a dwell time of $5 \mathrm{~s}$ per step. The data acquisition time of these elemental maps was not optimized; instead, high resolution maps were acquired to demonstrate proof-of concept for the elemental identification of the surface particle. The X-ray fluorescence intensity of the element ROI is reflected in the color coding of the 2D elemental maps. Maps were recorded at the surface of the sample (Figs. 5A, 5D, and 5G) as well as $25 \mu \mathrm{m}$ below the surface of the sample (Figs. 5B, 5E, and 5H) and $50 \mu \mathrm{m}$ below the surface of the sample (Figs. 5C, 5F, and 5I). The W L $\alpha$ X-ray fluorescent signal of Particle A's gold-plated tungsten indicator wire is clearly seen in Figure 5A and decreases in intensity as the sample is raised $25 \mu \mathrm{m}$ (Fig. 5B) and $50 \mu \mathrm{m}$ (Fig. 5C). As noted in Table 1 , the $\mathrm{Cu} \mathrm{K} \alpha$ energy ROI and $\mathrm{Cu} \mathrm{K} \beta$ energy ROI overlap with the W L $\alpha$ energy ROI. The Cu/W ROI overlap, along with the presence of $\mathrm{Cu}$ at this location in the mineral sample, results in the unavoidable appearance of $\mathrm{Cu}$ signal in Figures $5 \mathrm{~A}-5 \mathrm{C}$.

Figures 5D, 5E, and 5F are Pt La energy ROI maps, recorded in the same spatial locations as Figures 5A, 5B, and 5C, respectively. As can be seen in Figure 5D, a Pt ROI signal $(<140$ counts) appears near the tip of the indicator wire. As the sample is raised $25 \mu \mathrm{m}$ (Fig. 5E), the Pt signal not only increases in intensity but also in area. The Pt signal intensity and area increases further as the sample is raised $50 \mu \mathrm{m}$ (Fig. 5F). Also, in Figures 5D, 5E, and 5F, Au La signal can be seen (originating from the gold-plated tungsten indicator wire) due to the overlap of the Pt L $\alpha$ energy ROI and Au L $\alpha$ energy ROI overlap (Table 1). 
Figures 5G, 5H, and 5I show the Fe Ka signal from the same location of the Pt L $\alpha$ signal shown in Figures 5D, 5E, and 5F, respectively. As with the Pt signal discussed above, the Fe Ka signal increases in intensity and area as the sample is raised $25 \mu \mathrm{m}$ (Fig. 5H) and $50 \mu \mathrm{m}$ (Fig. 5I). This result confirms the presence of both $\mathrm{Pt}$ and $\mathrm{Fe}$ in the same spatial location, consistent with MLA results confirming the presence of a tetraferroplatinum particle.

While the spatial location and elemental information of Particle A has been obtained by MLA, the 2D elemental maps (Fig. 5A-5I) obtained by confocal MXRF indicate that a portion of Particle A is below the surface of the mineral sample. This observation is confirmed by microCT (Fig. 6), where it is clearly seen that the majority of the volume of Particle A extends $\sim 17 \mu \mathrm{m}$ into the surface of the mineral sample.

One limitation of the current confocal MXRF microscope operating software is that spectral information is not recorded during 2D scans. While the 2D Pt L $\alpha$ energy ROI maps presented in Figures 5D, 5E, and 5F indicate the presence of Pt within Particle A, spectral information is needed to confirm the presence of Pt within Particle A. The presence of Pt in Particle A was confirmed by moving the sample so that Particle A was centered within the confocal MXRF excitation/detection volume then recording a depth profile of the Pt L $\alpha$ energy ROI at this location (Fig. 7A). This depth profile was recorded using $3 \mu \mathrm{m}$ step sizes with a dwell time of $10 \mathrm{~s}$ per step. As can be seen from this depth profile, the Pt L $\alpha$ energy ROI signal begins to increase at approximately $20 \mu \mathrm{m}$ above the surface, which can be attributed to the difficultly of defining the "surface" of a sample using confocal MXRF. This difficulty arises from the geometry of the excitation/detection volume, which is the shape of a prolate spheroid. As the sample is raised, the edge of the excitation/detection volume comes into contact with the 
surface of the particle. The Pt L $\alpha$ energy ROI signal peaks at approximately $50 \mu \mathrm{m}$ below the surface before decreasing at approximately $110 \mu \mathrm{m}$ below the surface.

Having confirmed the presence of Particle A within the confocal MXRF excitation/detection volume, spectra were recorded as a function of depth to confirm the presence of Pt within Particle A. Figure 7B presents a series of spectra as a function of $\mathrm{Z}$ depth, recorded from $50 \mu \mathrm{m}$ above the surface to $100 \mu \mathrm{m}$ below the surface using $25 \mu \mathrm{m}$ steps and a dwell time of 100 s per step. As can be seen in Figure 7B, prominent peaks corresponding to Pt $\mathrm{L} \alpha$ (9.43 $\mathrm{keV})$ and Pt L $\beta(11.03 \mathrm{keV}) \mathrm{X}$-ray fluorescent energies are observed at depths of 25, 50 and 75 $\mu \mathrm{m}$ below the surface, confirming the presence of Pt within Particle A. No peaks corresponding to Pt are observed at $100 \mu \mathrm{m}$ below surface, indicating that the Pt L $\alpha$ energy ROI signal recorded at depths greater than $100 \mu \mathrm{m}$ below the surface in the depth profile (Fig. 7A) can be attributed to X-ray scattering from the epoxy puck within the Pt L $\alpha$ ROI.

An X direction (i.e., the direction parallel to the optics plane) line scan of the Pt L $\alpha$ energy ROI is provided in Figure 8A. The line scan was recorded at a depth of $50 \mu \mathrm{m}$ below the surface, with $3 \mu \mathrm{m}$ step sizes and a dwell time of $10 \mathrm{~s}$ per step. A confocal MXRF spectrum was recorded at an $\mathrm{X}$ position of $50 \mu \mathrm{m}$, presented in Figure $8 \mathrm{~B}$. The spectrum confirms not only the presence of Pt within Particle A, but also Cu (as seen in Figs. 5A, 5B, and 5C), Fe (as seen in Figs. 5G, 5H, and 5I), Cr, and Ni either within Particle A or the surrounding matrix.

\subsubsection{Embedded - Particle B}

Figure 9A presents a confocal MXRF 2D W L $\alpha$ energy ROI map of the gold-plated tungsten wire, placed to indicate the lateral (i.e., $\mathrm{X}$ and $\mathrm{Y}$ ) location of Particle B. The wire was placed in this location based on the spatial information of Particle B observed with micro-CT 
data (see Fig. 4). A magnified section of a reconstructed micro-CT slice, $\sim 300 \mu \mathrm{m}$ below the surface, is presented in Figure 9B. A high Z particle, corresponding to Particle B, can clearly be seen in the center of this slice. Figure $9 \mathrm{C}$ is a volume rendering of Particle B (illustrated in red) and surrounding mid-Z particles (illustrated in gray) within the region surrounding Particle B.

Figures 10A-10F are a series of confocal MXRF 2D elemental maps of Particle B. The 90 $\mu \mathrm{m}$ x $90 \mu \mathrm{m}$ maps, recorded in the region indicated by the white outlined box depicted in Figure 9A, were acquired using $15 \mu \mathrm{m}$ steps in the $\mathrm{X}$ and $\mathrm{Y}$ planes with a dwell time of $30 \mathrm{~s}$ per XY step and $25 \mu \mathrm{m}$ steps in the $\mathrm{Z}$ direction. Figures $10 \mathrm{~A}, 10 \mathrm{~B}$, and $10 \mathrm{C}$ are fluorescent intensity maps of the Pt L $\alpha$ energy ROI, recorded at $325 \mu \mathrm{m}$ (Fig. 10A), $350 \mu \mathrm{m}$ (Fig 10B), and $375 \mu \mathrm{m}$ (Fig. 10C) below the "surface" (here, the surface is referring to the point at which the maximum intensity of the gold-plated tungsten indicator wire is observed using confocal MXRF). As can be seen in Figure 10A, a small area emits a Pt L $\alpha$ energy ROI signal of roughly 18 counts. This region increases in area as the sample is raised $25 \mu \mathrm{m}$ (Fig. 10B), then decreases in area and intensity as the sample is raised to $375 \mu \mathrm{m}$ below the surface (Fig. 10C). Another significant elemental fluorescent signal is observed for the $\mathrm{Pb}$ L $\alpha$ energy ROI, as can be seen in Figures 10D, 10E, and 10F. The Pb L $\alpha$ energy ROI signal increases in both intensity and area when the sample is raised from $325 \mu \mathrm{m}$ below the surface (Fig. 10D) to $350 \mu \mathrm{m}$ below the surface (Fig. 10E). Raising the sample, so that the confocal MXRF excitation/detection volume is located 375 $\mu \mathrm{m}$ below the surface, results in a decrease in the $\mathrm{Pb}$ L $\alpha$ energy ROI signal, as seen in Figure $10 \mathrm{~F}$.

To confirm both the presence of $\mathrm{Pt}$ and $\mathrm{Pb}$ within Particle $\mathrm{B}$, a confocal MXRF spectrum was recorded at the location of Particle $\mathrm{B}$, at a distance of $350 \mu \mathrm{m}$ below the surface (corresponding to the location in Figures 10B and 10E). The spectrum, presented in Figure 11, 
was acquired using a $900 \mathrm{~s}$ dwell time. While the duration of the data acquisition is relatively long compared to the acquisition time of the spectra presented in Figure 7B, the resulting spectrum exhibits distinct peaks corresponding to Pt L $\alpha$ and Pt L $\beta$ X-ray fluorescent energies, as well as $\mathrm{Pb} \mathrm{L} \alpha$ and $\mathrm{Pb} \mathrm{L} \beta$ fluorescent $\mathrm{X}$-ray energies. These peaks confirm the presence of both $\mathrm{Pt}$ and $\mathrm{Pb}$ within Particle $\mathrm{B}$. In addition, $\mathrm{X}$-ray fluorescent peaks are observed for $\mathrm{Cr}, \mathrm{Fe}, \mathrm{Ni}$, and $\mathrm{Cu}$ within the location of Particle B.

\subsection{Confocal MXRF Detection Limits}

Using Particle B, the sensitivity of the confocal MXRF microscope in the analysis of highly heterogeneous samples can be approximated. The minimum detection limit (MDL) of the confocal MXRF microscope is obtained by calculating the standard deviation of Poisson statistics which is equal to the square root of the ROI intensity. It is the amount of analyte that gives a net fluorescence intensity which is three times the standard deviation of the background. Here, the amount could be concentration $(\%)$ for infinitely thick samples or mass $(\mathrm{g})$ for particulate samples. The correlative technique allows us to directly estimate the mass of particulates. This effort is done to provide a contextual discussion point for potential quantitative confocal MXRF measurements.

Assuming the mass concentration is given by $C_{i}$, the $\mathrm{MDL}_{i}$ can be defined using Equation 2 ,

$$
\mathrm{MDL}_{i}=\frac{3 \sigma_{B}}{I} \times C_{i}=\frac{3 \sqrt{I_{B}}}{I} x C_{i}
$$


where $I$ is the net intensity of the fluorescence energy ROI (for the Pt L $\alpha$ ROI, this is equal to 461 counts) and $\sigma_{B}$ is the standard deviation of the background. For an integration time of $900 \mathrm{~s}$, the upper limit of the MDL for a Pt particle (Particle B) is estimated to be $0.14 C_{i}$. The mass, $m_{i}$, of the analyte can be estimated from Equation 3.

$m_{i}=\rho_{i} \times V_{i} \times C_{i}$

Equation 3

The volume $\left(V_{i}\right)$ of the analyte can be obtained from the micro-CT data and, assuming that the element of interest is solely located within the particle, does not require the knowledge of the confocal MXRF excitation/detection volume. The volume of Particle B, measured using microCT, was estimated to be $3875 \mu \mathrm{m}^{3}$.The exact composition of Particle B is not known; however, if we assume a pure Pt particle with density $\left(\rho_{i}\right)$ of $21.5 \mathrm{~g} \mathrm{~cm}^{-3}$ and the volume of Particle B obtained by micro-CT, the mass of Pt is calculated to be $83 \mathrm{ng}$. Taking this value as $C_{i}$ and substituting into Equation 2 implies that the upper limit of the MDL is $11.6 \mathrm{ng}$ for Pt. This corresponds to a fully dense Pt particle with a volume of $541 \mu^{3}$ and equivalent diameter of $10.1 \mu \mathrm{m}$. Similarly, the assumption that the particle is tetraferroplatinum (with $\rho_{i}$ equal to $14.5 \mathrm{~g}$ $\mathrm{cm}^{-3}$ and $78 \%$ mass fraction of Pt) implies an upper limit of the minimum detection limit of 6.0 ng for Pt. Similar MDL calculations cannot be performed for the Fe content, since the Fe signal is also obtained from the surrounding matrix within the confocal excitation/detection volume.

If we assume that Particle B is pure $\mathrm{Pb}$, with $\rho_{i}$ equal to $11.34 \mathrm{~g} \mathrm{~cm}^{-3}$ the upper limit of the MDL for $\mathrm{Pb}$ is calculated to be $2.4 \mathrm{ng}$. This corresponds to a $\mathrm{Pb}$ particle with a volume of $215 \mu \mathrm{m}^{3}$ and equivalent diameter of $7.4 \mu \mathrm{m}$. 
As can be seen from the spectrum in Figure 11, several elements other than $\mathrm{Pt}$ and $\mathrm{Pb}$ are detected within the confocal excitation/detection volume surrounding Particle $\mathrm{B}$, such as $\mathrm{Fe}, \mathrm{Ni}$, $\mathrm{Cu}$, and $\mathrm{Cr}$. Without calibrated micro-CT data, individual particles containing these elements were not identified. However, a conservative upper limit for these elements can be calculated if we assume a confocal excitation/detection volume of $30 \mu \mathrm{m}$ x $30 \mu \mathrm{m}$ x $50 \mu \mathrm{m}\left(188,500 \mu^{3}\right)$ and assume that this volume is entirely fully dense for each detected element, $\mathrm{Fe}, \mathrm{Ni}, \mathrm{Cu}$ or $\mathrm{Cr}$. The confocal MXRF excitation/detection volume will certainly change with the X-ray fluorescence energy; however, for discussion purposes this volume will be used to calculate conservative upper limits of the MDL for each element. With these assumptions, the calculated minimum detection limits of these elements are displayed in Table 2. Of note is the calculated MDL for Fe at $51.3 \mathrm{ng}$, which arises from the intensity of the Fe K $\alpha$ peak observed in Figure 11. These first order approximations demonstrate that this correlative technique possesses a micrometer level sensitivity and nanogram level MDL in a relatively short acquisition time of $15 \mathrm{~min}$.

Several caveats must be understood though when approximating an MDL for confocal MXRF and may not be directly comparable from one instrument to the next. 1) The signal from subsurface particles will be reduced by the overlying material. Even a low density aerogel exhibits this phenomenon.[25] Based upon our first order estimates given above, the MDL will be even lower for particles closer to the surface. 2) The excitation energy of the X-ray tube will affect the efficiency in which the analyte will fluoresce; X-ray tubes of differing anode material will produce different excitation efficiencies. 3) Instrument geometry will affect depth of signal measurements. The confocal MXRF instrument highlighted here has optics positioned $30^{\circ}$ from the surface normal. An instrument with $45^{\circ}$ optics will have a lower sensitivity at depth due to a longer path length to the surface of the sample. 4) The polycapillary optics act as a low pass 
energy filter. The energy transmission of the optics used in this study is reduced above $\sim 15 \mathrm{keV}$. Finally, 5) the conservative estimations of the MDLs are based on counting statistics. That is, the net intensity of an element's fluorescent signal is considered and directly affects the estimation of the MDL (see Eqn. 2); therefore, the MDL estimation should be considered spectrum-specific.

\section{Conclusions and Future Outlook}

We have demonstrated that it is possible to non-destructively detect and elementally identify high $\mathrm{Z}$ particles of interest, up to $\sim 300-350 \mu \mathrm{m}$ below the surface, by integrating laboratory-based micro X-ray computed tomography for rapid location and laboratory-based confocal micro X-ray fluorescence spectroscopy for rapid elemental identification. Micro-CT allows the identification of the spatial locations of high $\mathrm{Z}$ particles within a low $\mathrm{Z}$ matrix with micrometer level resolution, non-destructively, by simply identifying localized regions of high X-ray attenuation. The confocal MXRF microscope allows these particles of interest to be analyzed for elemental composition. A significant aspect of this demonstration is the confocal MXRF instrument itself, which allows for spatial discrimination of elemental fluorescent signals, at significant depths beneath the surface of a sample.

We have also provided a method for determining conservative estimates of minimum detection limits. By stating several assumptions, we have used data from confocal MXRF spectra and from micro-CT tomograms to provide upper limits on the sensitivity that can be expected from this particular analysis. A more rigorous approach would be to validate this technique using standard materials, which could be the topic for a follow-up study.

The confocal MXRF microscope used in this work utilized an Ag source and a Si-PIN photodiode Amptek detector with a confocal volume of approximately $30 \mu \mathrm{m}$ x $30 \mu \mathrm{m}$ x $50 \mu \mathrm{m}$. 
However, significant progress has been made in both polycapillary and X-ray detector technology. Currently, confocal excitation/detection volumes of $\sim 20 \mu \mathrm{m} \times 20 \mu \mathrm{m} \times 20 \mu \mathrm{m}$ and below[26] can be routinely achieved which increases the flux density at the sample. For detection of fluorescence X-rays between $6 \mathrm{keV}$ to12 keV, the utilization of a Mo source would be more useful than a Ag source. The Mo lines are more efficiently generated in the source X-ray tube for a given applied voltage and are more efficiently absorbed by either K or L edges of elements of interest which leads to higher fluorescence intensities. Additionally, Mo sources can be operated at higher power $(50 \mathrm{~W})$, when compared to Ag sources $(25 \mathrm{~W})$, leading to higher flux and shorter integration times for equivalent statistics. State-of-the-art silicon drift detectors would also lead to shorter integration times, compared to a Si-PIN photodiode detector. Combining these, it is estimated that over an order of magnitude improvement in throughput can be obtained ( $15 \mathrm{X}$ to $25 \mathrm{X})$ depending on the sample and the depth of the particle studied. Such a large improvement is expected to allow for detection of elements of mass of $\sim 100 \mathrm{pg}(\sim 2 \mu \mathrm{m}$ particle of $\mathrm{Pb}$ ) or better for reasonable integration times of about one hour. For particles closer to the surface, picogram level sensitivities can be expected.

For this study, it was imperative that fiducial markers be placed on the sample surface, to indicate the locations of the particles of interest. This aided in reducing the amount of time needed to locate the particles using the confocal MXRF microscope, which otherwise would have required a complete $3 \mathrm{D}$ rastering of the sample. The time required to locate and analyze particles could further be reduced by fully integrating micro-CT and confocal MXRF into a single instrument. By using universal coordinate axes, a sample could be imaged using microCT, particles of interest identified, and then the sample could be moved into the correct position to allow for confocal MXRF elemental analysis. 
Modern micro-CT instruments routinely achieve sub-micrometer resolution for samples a few millimeters in size. The technique enables the sampling of a relatively large three dimensional volume in a matter of a few hours to locate all the grains of interest based on gray levels representing differing attenuation of X-rays within the voxel. With proper CT calibration, many of the grains of interest can be determined. However, in other R\&D applications where there is no prior knowledge of the compounds present in a heterogeneous sample, proper CT calibration might require polished cross-sections of samples measured using SEM-EDS to be correlated to X-ray CT sections. The micro-CT/confocal MXRF correlative technique provides the capability to measure the elemental make-up of different grains of the sample directly.

For mining applications, MLA can provide very high spatial resolution information and elemental composition of particles of interest at the surface, whereas high resolution X-ray CT technology can overcome many of the problems inherent to MLA and play a very important complementary role. This technique enables collection of statistically relevant data with very short turn-around time. With the unambiguous, isotropic information content of X-ray CT, true 3D data of individual particles is collected to completely describe the liberation state and particle association, which is important for accurate predictions of the grade recovery.

The coupling of high resolution X-ray CT with confocal MXRF also helps mitigate and overcome another inherent limitation of the confocal MXRF technique on sample sizes arising from limited X-ray penetration into samples and absorption of emitted fluorescence X-rays in the sample. For CT grayscale calibration, probing of points deep inside the sample is often not required and limited probing of selected points at and near the surface of the specimen is sufficient. 
In this study, we chose to analyze mineralogical samples containing high $\mathrm{Z}$ particles in a low $\mathrm{Z}$ matrix: the deepest particle probed was $\sim 350 \mu \mathrm{m}$ from the surface. Extrapolation of the data suggests that particles up to $\sim 750 \mu \mathrm{m}$ deep can easily be studied. Depending upon the packing fraction of the particles, this implies a sample of $\sim 1.5$ to $4 \mathrm{~mm}$ in diameter. This is made possible due to matrix absorption of fluorescent X-rays of interest being very small. For biological samples composed of much lower densities, up to $10 \mathrm{~mm}$ diameter samples could be studied.

This methodology can easily be extended to include the analyses of samples containing high $\mathrm{Z}$ particles in a high $\mathrm{Z}$ matrix and samples with low $\mathrm{Z}$ particles in low $\mathrm{Z}$ matrix. In these cases, high absorption of fluorescent X-rays of interest by the matrix may limit the probing depths within the samples, sometimes significantly. In such cases, one would require particles of interest to be present at or near the surface for confocal MXRF analysis. The study of samples containing low $\mathrm{Z}$ particles in a low $\mathrm{Z}$ matrix imposes an additional constraint in that the experiment must be conducted in vacuum (unlike air for the current study) to mitigate the absorption of fluorescent X-rays in air. This is easily achieved and such an instrument is currently being designed at Los Alamos National Laboratory.

This non-destructive analysis at depth, which cannot be accomplished with either SEMbased EDS or with conventional MXRF, shows significant promise for the characterization of samples embedded in other interesting environments, such as actinides or toxic elements within soil samples. This study has shown that this correlative technique can open new avenues of research in the fields of materials science, biology, toxicology and mining, to name a few.

\section{Acknowledgements}


This work was partially funded by National Science Foundation (SS \& MF), under the award number NSF IIP 1248744. Los Alamos National Laboratory is operated by Los Alamos National Security LLC under contract number DE-AC52-06NA25396 for the US Department of Energy.

\section{REFERENCES}

[1] Z. Yu, P. Robinson, P. McGoldrick, An Evaluation of Methods for the Chemical Decomposition of Geological Materials for Trace Element Determination using ICP-MS, Geostandards Newsletter, 25 (2001) 199-217.

[2] S. F. Durrant, Laser ablation inductively coupled plasma mass spectrometry: achievements, problems, prospects, J. Anal. At. Spectrom., 14 (1999) 1385-1403.

[3] A.A. Ammann, Inductively coupled plasma mass spectrometry (ICP MS): a versatile tool, J. Mass Spectrom., 42 (2007) 419-427.

[4] R. Fandrich, Y. Gu, D. Burrows, K. Moeller, Modern SEM-based mineral liberation analysis, Int. J. Miner. Process., 84 (2007) 310-320.

[5] B. Kanngießer, I. Mantouvalou, W. Malzer, T. Wolff, O. Hahn, Non-destructive, depth resolved investigation of corrosion layers of historical glass objects by 3D Micro X-ray fluorescence analysis, J. Anal. At. Spectrom., 23 (2008) 814-819.

[6] I. Mantouvalou, K. Lange, T. Wolff, D. Grotzsch, L. Luhl, M. Haschke, O. Hahn, B. Kanngießer, A compact 3D micro X-ray fluorescence spectrometer with X-ray tube excitation for archaeometric applications, J. Anal. At. Spectrom., 25 (2010) 554-561.

[7] I. Mantouvalou, W. Malzer, B. Kanngießer, Quantification for 3D micro X-ray fluorescence, Spectrochim. Acta Part B, 77 (2012) 9-18.

[8] I. Mantouvalou, W. Malzer, I. Schaumann, L. Luhl, R. Dargel, C. Vogt, B. Kanngiesser, Reconstruction of Thickness and Composition of Stratified Materials by Means of 3D Micro Xray Fluorescence Spectroscopy, Anal. Chem., 80 (2008) 819-826.

[9] I. Mantouvalou, T. Wolff, O. Hahn, I. Rabin, L. Lühl, M. Pagels, W. Malzer, B. Kanngiesser, 3D Micro-XRF for Cultural Heritage Objects: New Analysis Strategies for the Investigation of the Dead Sea Scrolls, Anal. Chem., 83 (2011) 6308-6315.

[10] I. Schaumann, W. Malzer, I. Mantouvalou, L. Lühl, B. Kanngießer, R. Dargel, U. Giese, C. Vogt, Preparation and characterization of polymer layer systems for validation of 3D Micro Xray fluorescence spectroscopy, Spectrochim. Acta Part B, 64 (2009) 334-340.

[11] W. Malzer, B. Kanngießer, A model for the confocal volume of 3D micro X-ray fluorescence spectrometer, Spectrochim. Acta Part B, 60 (2005) 1334-1341.

[12] S. Smolek, B. Pemmer, M. Folser, C. Streli, P. Wobrauschek, Confocal micro-x-ray fluorescence spectrometer for light element analysis, Rev. Sci. Instrum., 83 (2012) 083703083706.

[13] B. Kanngießer, W. Malzer, A.F. Rodriguez, I. Reiche, Three-dimensional micro-XRF investigations of paint layers with a tabletop setup, Spectrochim. Acta Part B, 60 (2005) 41-47. [14] B.M. Patterson, K.A. Obrey, G.J. Havrilla, Further Characterizations of Sputtered Copper Beryllium Capsules Using Confocal Micro X-Ray Fluorescence, Fusion Sci. Technol., 59 (2011) 121-125. 
[15] B.M. Patterson, K.A.D. Obrey, G.J. Havrilla, Nondestructive Investigations of a Copperand Argon-Doped Sputtered Beryllium Capsule Using X-Rays in Three Dimensions, Fusion Sci. Technol., 55 (2009) 417-423.

[16] M. Menzel, A. Schlifke, M. Falk, J. Janek, M. Fröba, U.E.A. Fittschen, Surface and in-depth characterization of lithium-ion battery cathodes at different cycle states using confocal micro-Xray fluorescence-X-ray absorption near edge structure analysis, Spectrochim. Acta Part B, 85 (2013) 62-70.

[17] R.A. Ketcham, W.D. Carlson, Acquisition, optimization and interpretation of X-ray computed tomographic imagery: Applications to the geosciences., Computers and Geosciences, 27 (2001) 381-400.

[18] B. Patterson, K. Henderson, Z. Smith, Measure of morphological and performance properties in polymeric silicone foams by X-ray tomography, J. Mater. Sci., 48 (2013) 19861996.

[19] B.M. Patterson, J.P. Escobedo-Diaz, D. Dennis-Koller, E. Cerreta, Dimensional Quantification of Embedded Voids or Objects in Three Dimensions Using X-Ray Tomography, Microsc. Microanal., 18 (2012) 390-398.

[20] P.C.J. Donoghue, S. Bengtson, X.-p. Dong, N.J. Gostling, T. Huldtgren, J.A. Cunningham, C. Yin, Z. Yue, F. Peng, M. Stampanoni, Synchrotron X-ray tomographic microscopy of fossil embryos, Nature, 442 (2006) 680-683.

[21] J.D. Miller, C.L. Lin, C. Garcia, H. Arias, Ultimate recovery in heap leaching operations as established from mineral exposure analysis by X-ray microtomography, Int. J. Miner. Process., 72 (2003) 331-340.

[22] J.D. Miller, C.L. Lin, Three-dimensional analysis of particulates in mineral processing systems by cone beam X-ray microtomography, Minerals \& Metallurgical Processing, 21 (2004) 113.

[23] J.D. Miller, C.L. Lin, L. Hupka, M.I. Al-Wakeel, Liberation-limited grade/recovery curves from X-ray micro CT analysis of feed material for the evaluation of separation efficiency, Int. J. Miner. Process., 93 (2009) 48-53.

[24] Y. Gu, Automated Scanning Electron Microscope Based Mineral Liberation Analysis, Journal of Minerals \& Materials Characterization \& Engineering, 2 (2003) 33-41.

[25] B.M. Patterson, K.A. DeFriend Obrey, C.E. Hamilton, G.J. Havrilla, Three-dimensional density measurements of ultra low density materials by X-ray scatter using confocal micro X-ray fluorescence spectroscopy, X-Ray Spectrom., 41 (2012) 253-258.

[26] K. Tsuji, K. Nakano, Development of a new confocal 3D-XRF instrument with an X-ray tube, J. Anal. At. Spectrom., 26 (2011) 305-309. 


\section{FIGURE CAPTIONS}

Figure 1. Schematic of the confocal micro X-ray fluorescence microscope. Source X-rays (blue) are focused onto the sample using a polycapillary focusing optic Detected X-rays (red) travel through a polycapillary optic that is placed on the detector. A significant portion of fluorescent $\mathrm{X}$-rays (green) do not reach the detector.

Figure 2: Samples of mineral particles containing $\mathrm{Pt}$ and $\mathrm{Pb}$ bearing minerals embedded in epoxy. These samples were imaged using an Xradia MicroXCT prior to embedding them in a larger epoxy puck.

Figure 3. SEM micrographs of a mineral sample and gold-plated tungsten wires indicating the lateral and horizontal position of Particle A (A) and the lateral position of the subsurface Particle B (B). Particle B is $\sim 300 \mu \mathrm{m}$ below the surface.

Figure 4. Micro X-ray computed tomographic reconstructed slices of the mineral sample, highlighting the surface location of Particle A and subsurface location of Particle B. Axes are in millimeters.

Figure 5. Confocal MXRF 2D fluorescent intensity maps of W L $\alpha$ energy ROI (A-C), Pt L $\alpha$ energy ROI (D-F), and Fe Ka energy ROI (G-I) of Particle A. Maps were acquired at the surface (A, D, and G), 25 $\mu \mathrm{m}$ below the surface (B, E, and $\mathrm{H}$ ), and $50 \mu \mathrm{m}$ below the surface (C, F, and I). Map dimensions are 250 $\mu \mathrm{m} \times 400 \mu \mathrm{m}$. Maps were acquired by using $3 \mu \mathrm{m}$ steps in the $\mathrm{X}$ and $\mathrm{Y}$ directions and a dwell time of $5 \mathrm{~s}$ per step. $\mathrm{Cu}$ signal can be seen in $\mathrm{B}$ and $\mathrm{C}$, due to overlap of the $\mathrm{Cu} \mathrm{K} \alpha$ and $\mathrm{K} \beta$ energy ROIs with $\mathrm{W} \mathrm{L} \alpha$ energy ROI (see Table 1); additionally, a Au signal can be seen in D-F due to overlap of the Au La with Pt L $\alpha$ energy ROIs. This highlights the need for spectral imaging when generating 2D confocal MXRF maps.

Figure 6. Micro-CT reconstructed slices and volume rendering (red) of the region surrounding Particle A, highlighting that the majority of the particle is located beneath the surface. Inset: A micro-CT reconstructed slice of the region surrounding Particle A (red), as viewed from the top-down.

Figure 7. A) A confocal MXRF depth profile of Pt L $\alpha$ ROI signal using $3 \mu \mathrm{m}$ steps in the $\mathrm{Z}$ direction with a dwell time of $10 \mathrm{~s}$ per step for Particle A. B) Confocal MXRF spectra of Particle $A$ as a function of $Z$ depth. Spectra were acquired by raising the sample towards the polycapillary optics ( $Z$ direction) using a $25 \mu \mathrm{m}$ step size and a $100 \mathrm{~s}$ dwell time per step. Signal from the sample begins at a $Z$ depth of $-25 \mu \mathrm{m}$ due to the confocal MXRF excitation/detection volume at the edge of the surface. A peak at $9.43 \mathrm{keV}$, corresponding to Pt L $\alpha$, emerges at a $\mathrm{Z}$ depth of $0 \mu \mathrm{m}$ and becomes more prominent at $Z$ depths of 25 and $50 \mu \mathrm{m}$, along with the emergence of a peak at $11.03 \mathrm{keV}$, corresponding to $\mathrm{Pt} \mathrm{L} \beta$, at these depths. Both peaks begin to diminish at a $Z$ depth of $75 \mu \mathrm{m}$, with no signal being observed $100 \mu \mathrm{m}$ below the surface. 
Figure 8. A) A confocal MXRF line scan of Particle A, showing the Pt L $\alpha$ ROI signal. The sample was scanned in the $\mathrm{X}$ direction (using $3 \mu \mathrm{m}$ steps at a dwell time of $10 \mathrm{~s}$ per step) by moving the sample in the direction parallel to the polycapillary optics at a constant $\mathrm{Z}$ depth of 50 $\mu \mathrm{m}$. B) Confocal MXRF spectrum of Particle A at an X position of $50 \mu \mathrm{m}$.

Figure 9. A) A confocal MXRF 2D fluorescent intensity map of the gold-plated tungsten wire (W L $\alpha$ energy ROI), indicating the lateral position of Particle B as viewed from the surface. The wire was placed approximately $49 \mu \mathrm{m}$ from the lateral position of Particle B, on the surface of the mineral sample. This map was acquired over a $300 \mu \mathrm{m}$ x $300 \mu \mathrm{m}$ area using $30 \mu \mathrm{m}$ steps with a dwell time of $1 \mathrm{~s}$ per step. Once the gold-plated tungsten indicator wire was located using the confocal MXRF microscope, the sample was raised $300 \mu \mathrm{m}$ and scanned in the region indicated by the white outlined area. B) A reconstructed micro-CT slice (top -down view) of the mineral sample at a depth of $\sim 300 \mu \mathrm{m}$ below the region is shown in Fig. 9A. The high $\mathrm{Z}$ Particle B appears as a concentrated region of high grayscale values. C) A volume rendering of Particle B (red) and surrounding particles (gray). Confocal MXRF 2D maps of this area are presented in Figures 10A-10F.

Figure 10. Confocal MXRF 2D fluorescent intensity maps of Particle B. Maps are plots of the intensity (counts) of a specified energy ROI; Pt L $\alpha$ energy ROI (A-C) and Pb L $\alpha$ energy ROI (DF). Maps were acquired $325 \mu \mathrm{m}$ below the surface (A and D), $350 \mu \mathrm{m}$ below the surface (B and $\mathrm{E}$ ), and $375 \mu \mathrm{m}$ below the surface (C and F). Map dimensions are $90 \mu \mathrm{m}$ x $90 \mu \mathrm{m}$. Maps were acquired by using $15 \mu \mathrm{m}$ steps in the $\mathrm{X}$ and $\mathrm{Y}$ directions and a dwell time of $30 \mathrm{~s}$ per step.

Figure 11. A confocal MXRF spectrum of Particle B, $350 \mu \mathrm{m}$ below the surface (i.e., below the location of gold-plated tungsten indicator wire). A dwell time of $900 \mathrm{~s}$ was used to provide sufficient signal to discern peaks for both the $\mathrm{Pb}$ and $\mathrm{Pt}$ fluorescent peaks. 


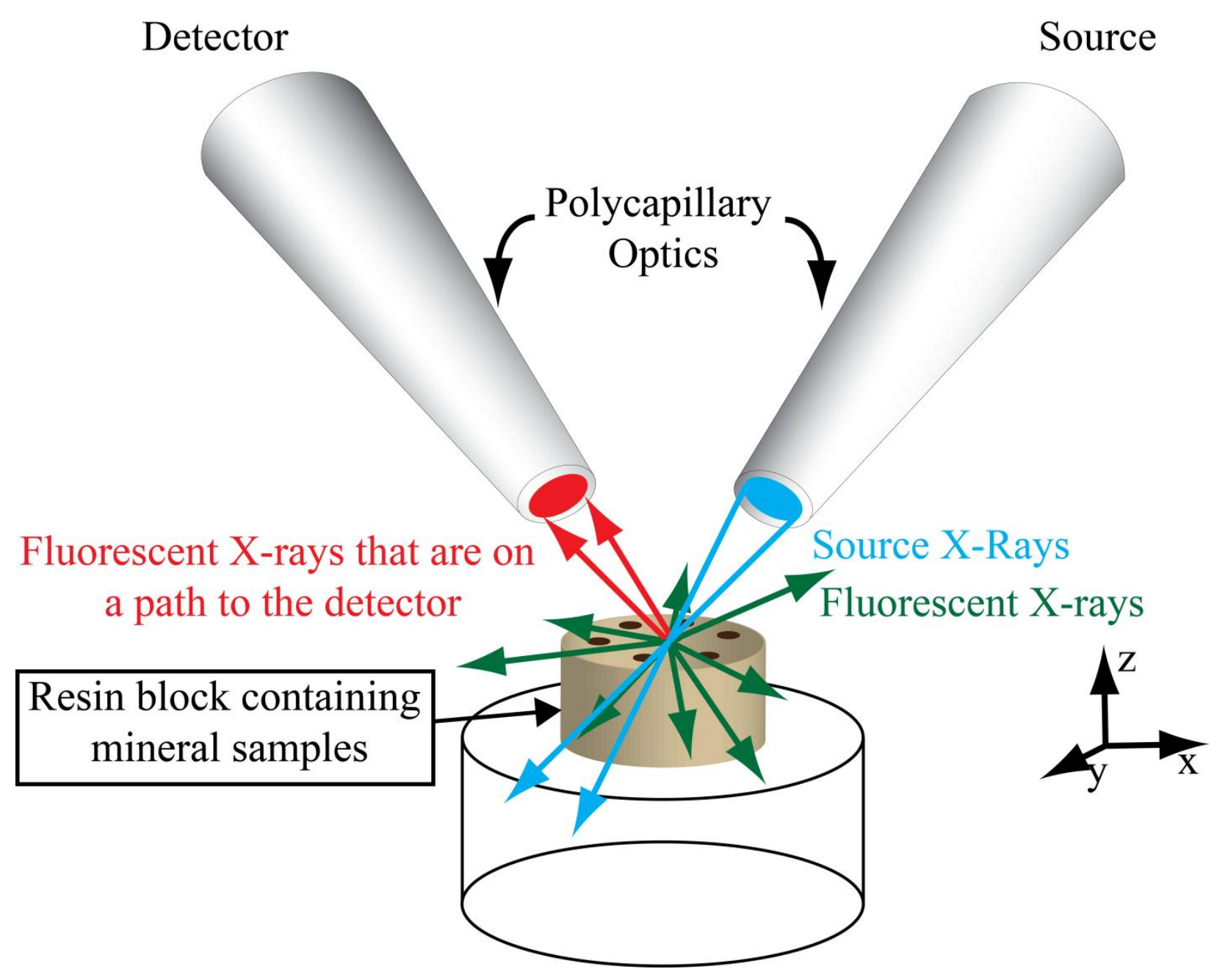

Figure 1 


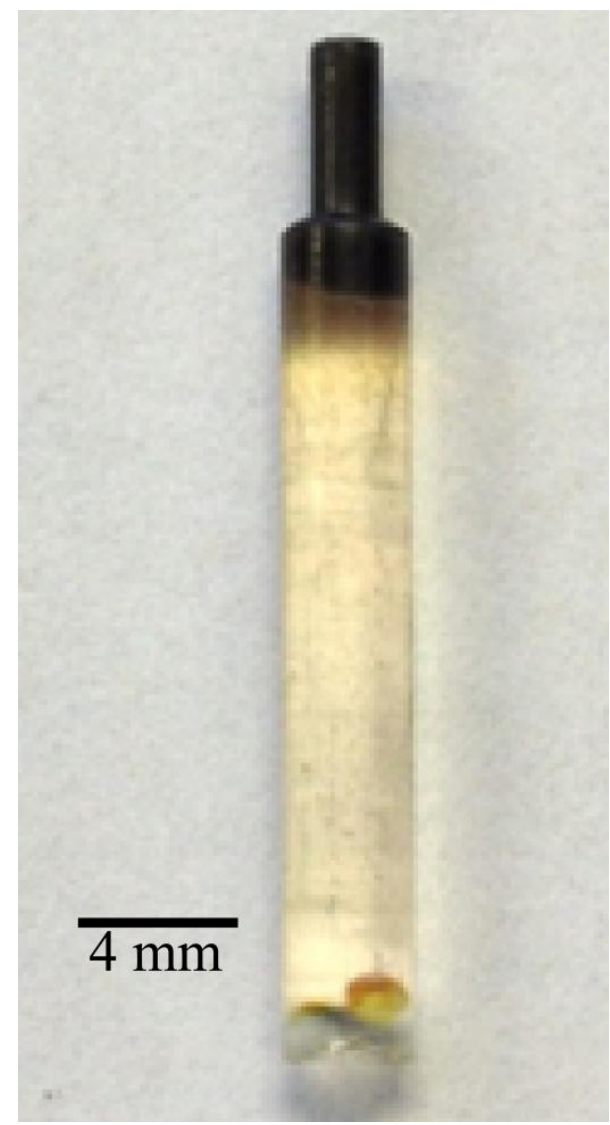

Figure 2 


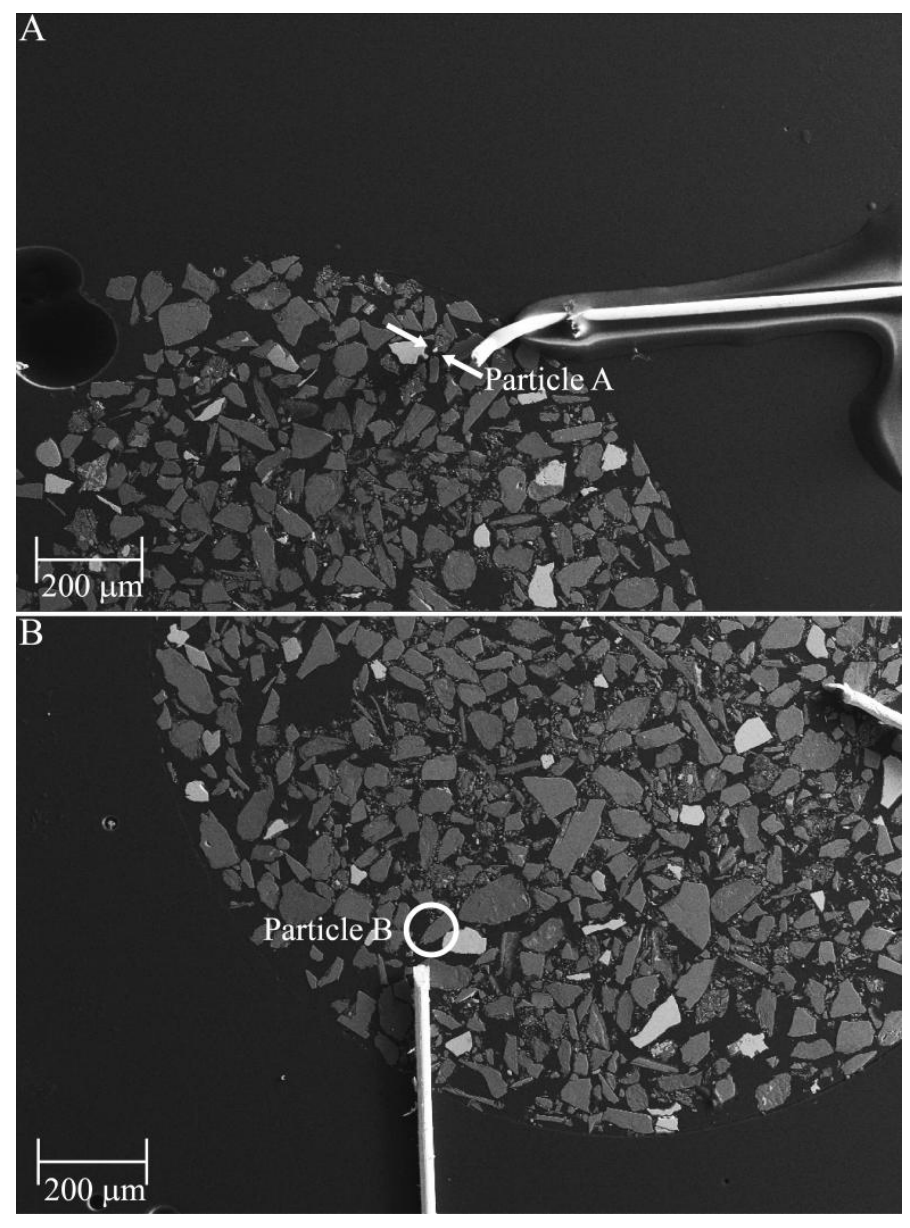

Figure 3 


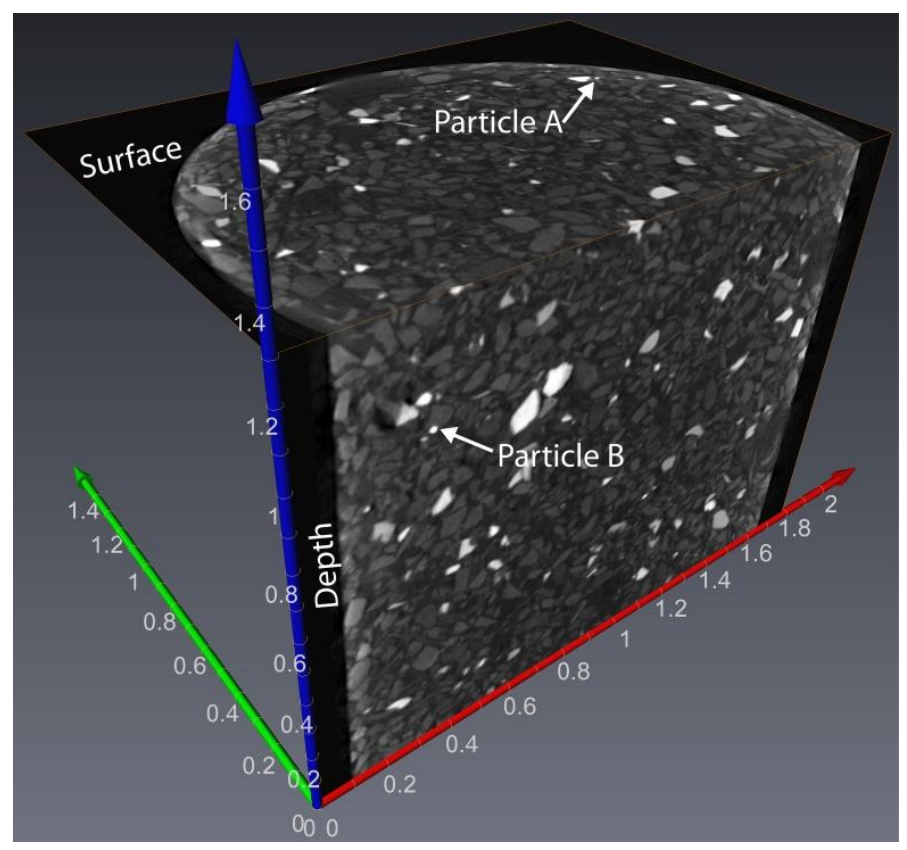

Figure 4

29 


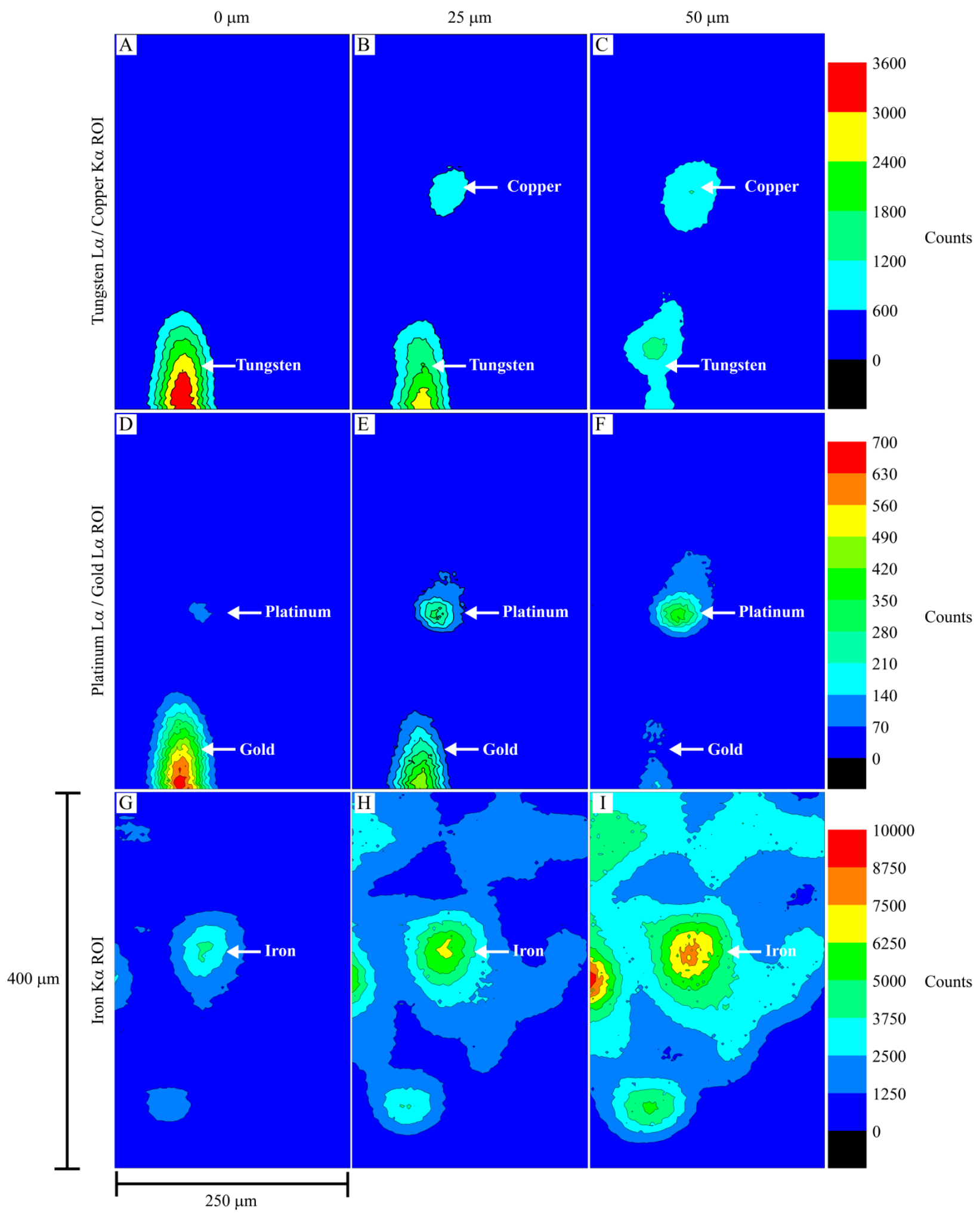

Figure 5 


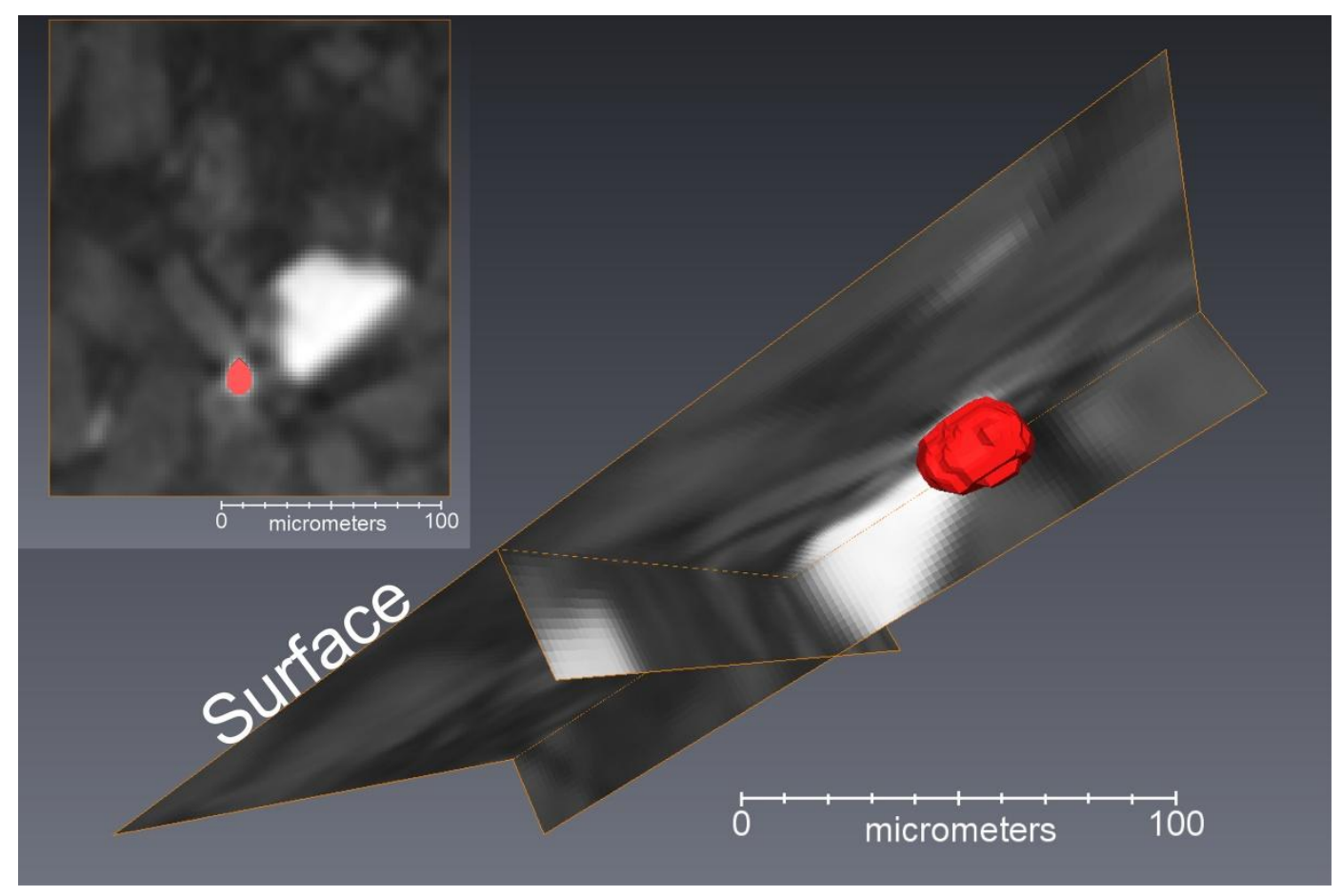

Figure 6 


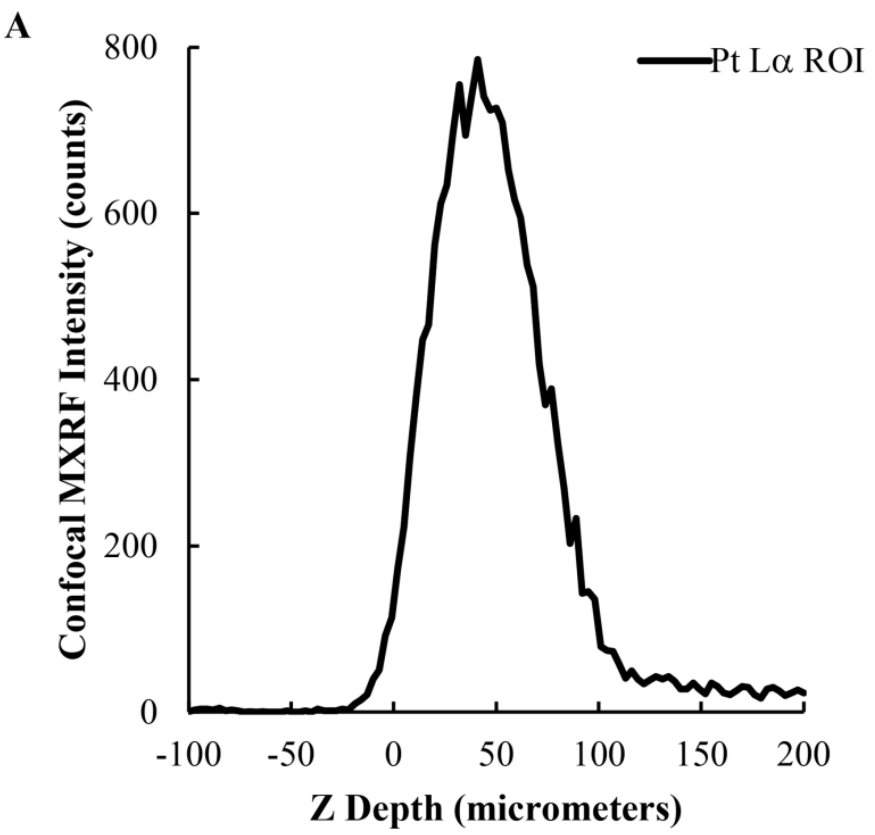

B

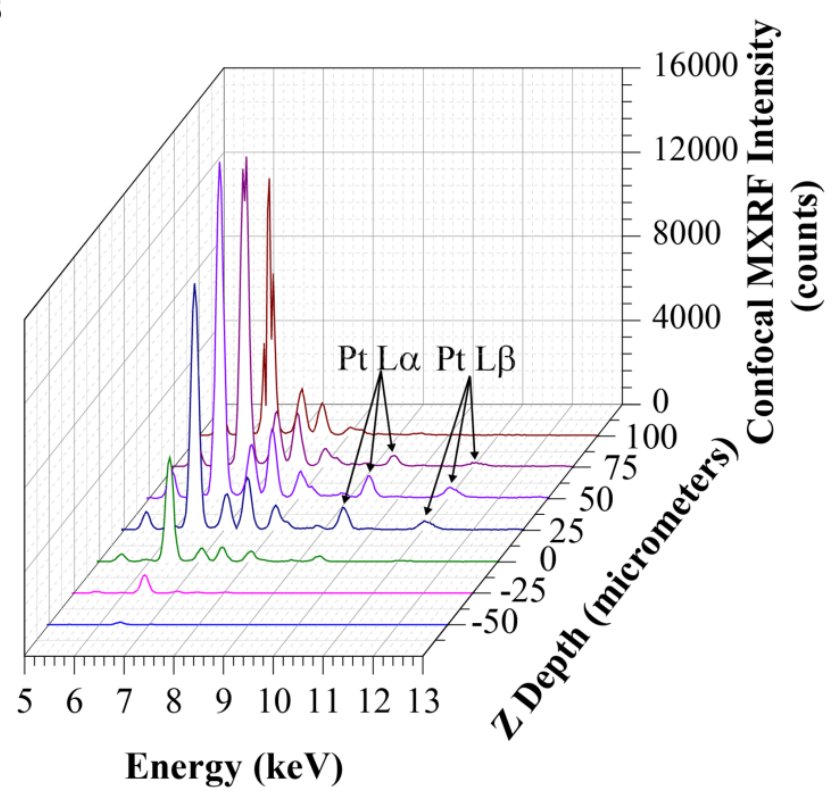

Figure 7 

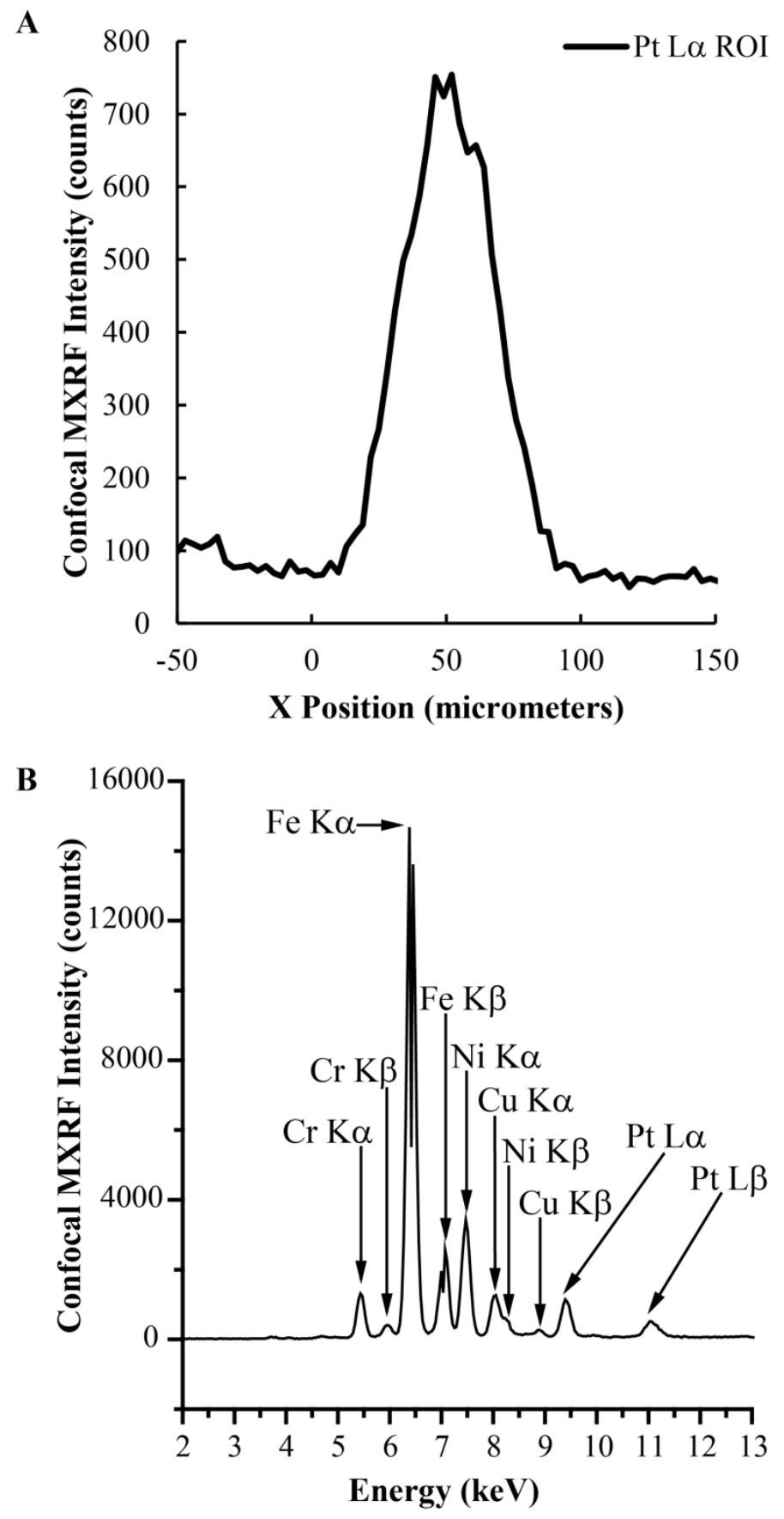

Figure 8 


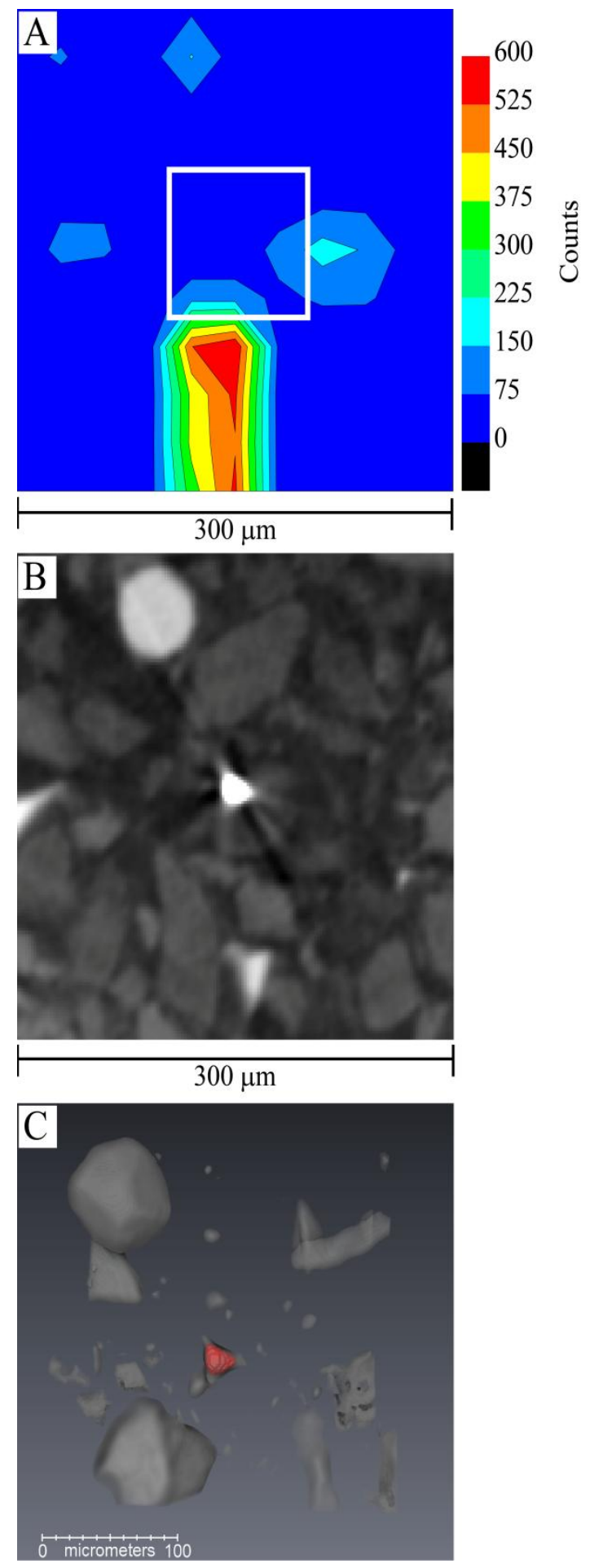

Figure 9 
$325 \mu \mathrm{m}$
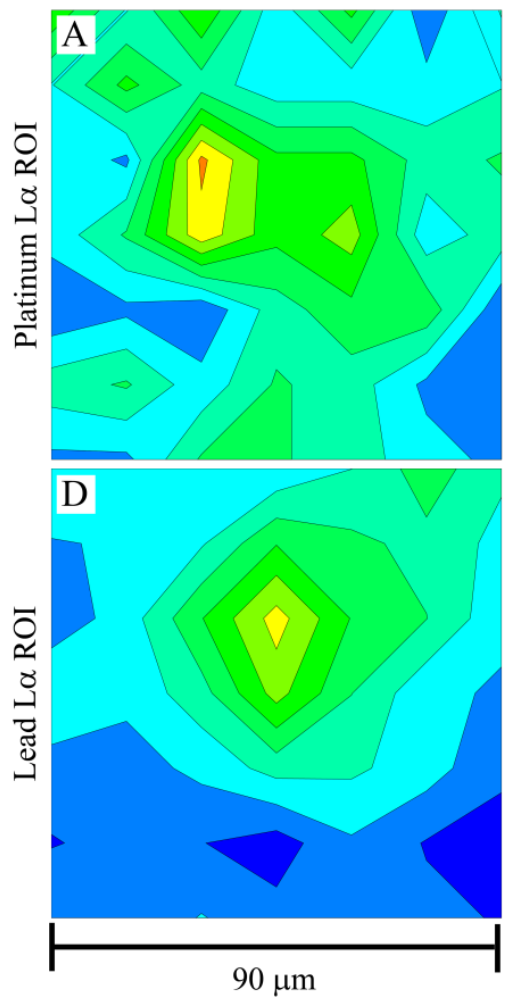

$350 \mu \mathrm{m}$
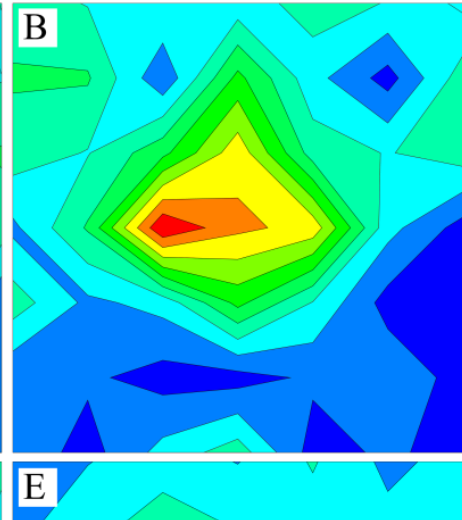
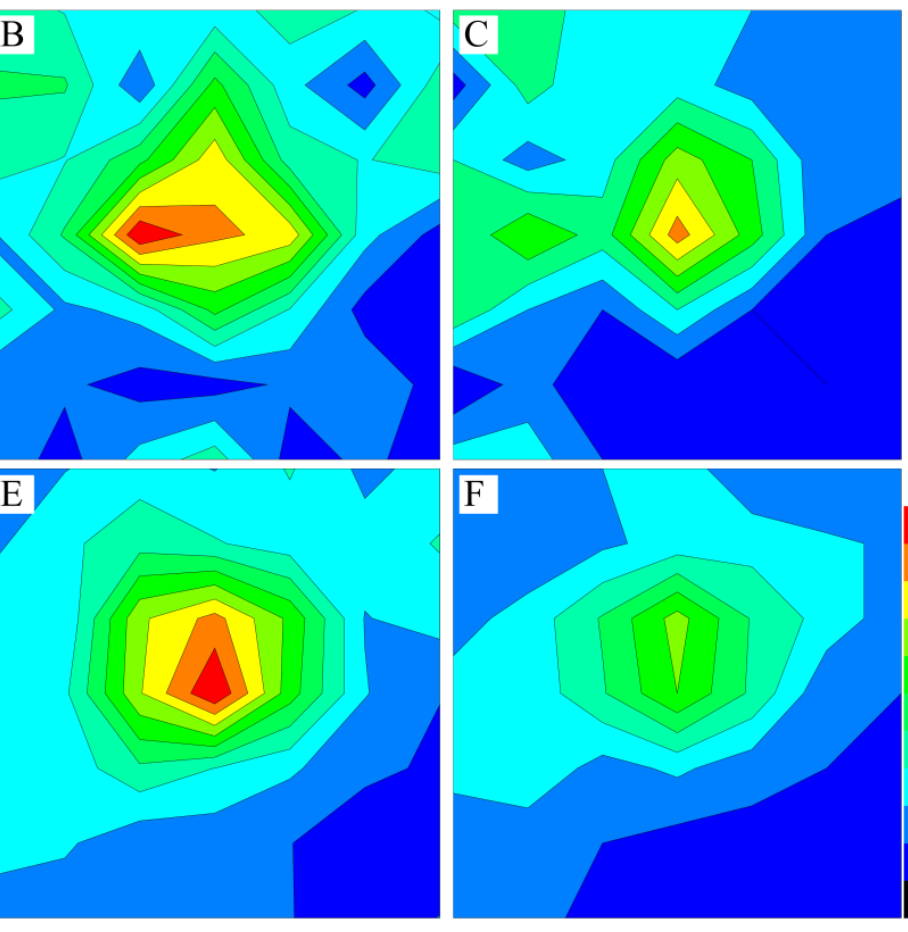

18
16
14

12

10

8 Counts

F

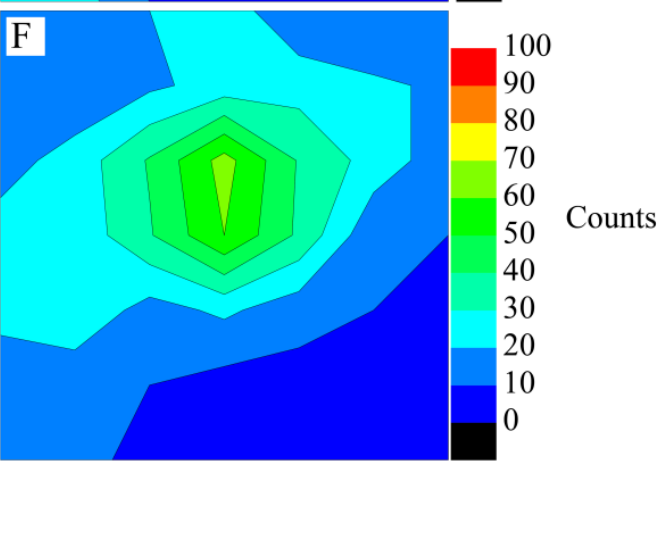

Figure 10 


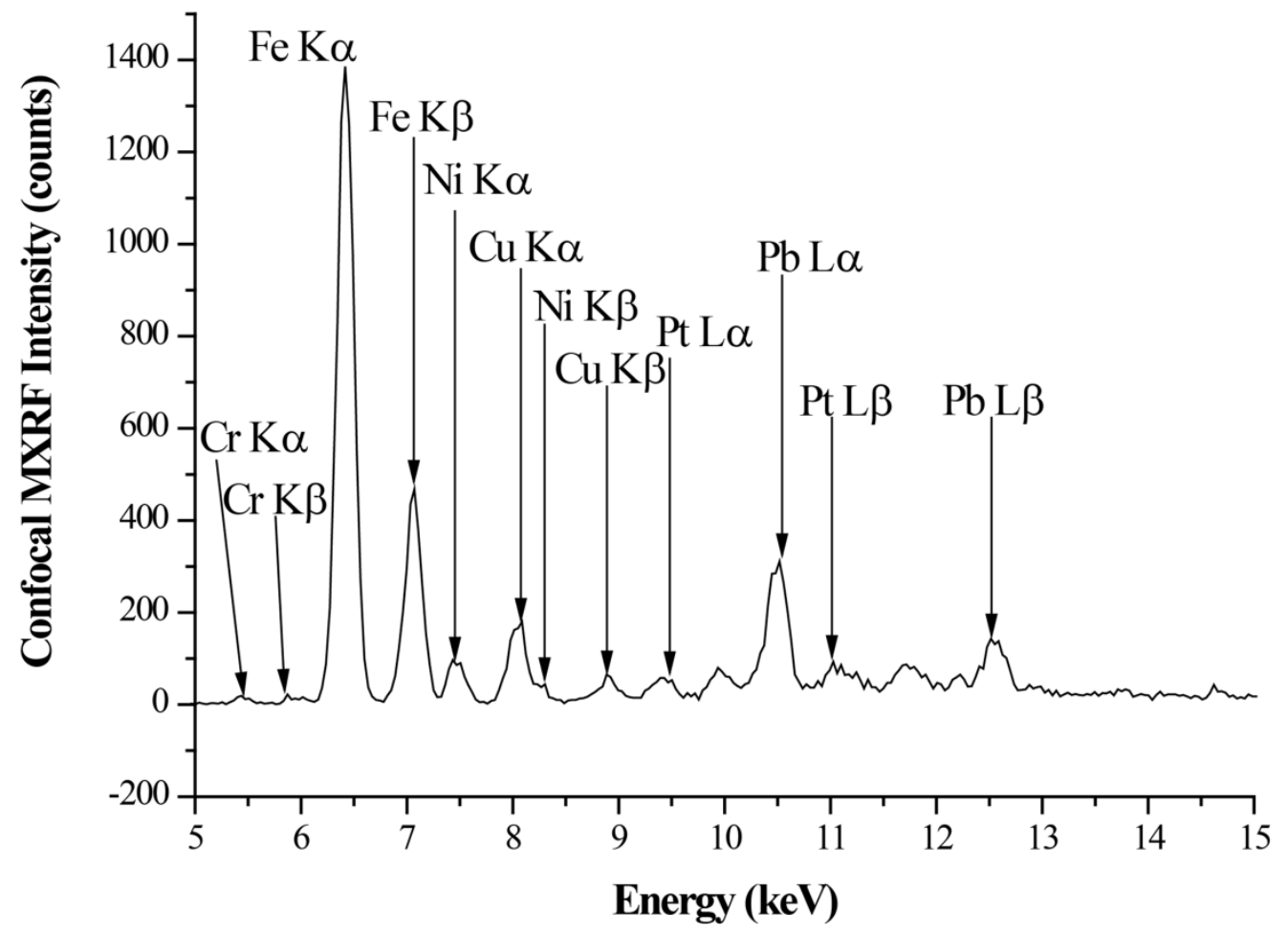

Figure 11 
Table 1. Energy regions of interest collected using confocal MXRF.

\begin{tabular}{|c|c|c|c|}
\hline Element & Line & Energy $(\mathrm{keV})$ & Energy Range (keV) \\
\hline Chromium & $\mathrm{K} \alpha$ & 5.411 & 5.33 to 5.62 \\
\hline Nickel & $\mathrm{K} \alpha$ & 7.472 & 7.29 to 7.72 \\
\hline \multirow[t]{2}{*}{ Iron } & $\mathrm{K} \alpha$ & 6.400 & 6.16 to 6.63 \\
\hline & $\mathrm{K} \beta$ & 7.059 & 6.74 to 7.25 \\
\hline \multirow[t]{2}{*}{ Copper $^{a}$} & $\mathrm{~K} \alpha$ & 8.041 & 7.76 to $8.34^{a}$ \\
\hline & $\mathrm{K} \beta$ & 8.907 & 8.63 to 9.07 \\
\hline Tungsten $^{a}$ & $\mathrm{~L} \alpha$ & 8.396 & 7.98 to $8.70^{a}$ \\
\hline \multirow[t]{2}{*}{ Gold $^{b}$} & $\mathrm{~L} \alpha$ & 9.711 & 9.32 to $10.32^{b}$ \\
\hline & $\mathrm{L} \beta$ & 11.582 & 10.99 to 11.86 \\
\hline \multirow[t]{2}{*}{ Platinum } & $\mathrm{L} \alpha$ & 9.441 & 9.21 to $9.61^{b}$ \\
\hline & $\mathrm{L} \beta$ & 11.249 & 10.63 to 11.61 \\
\hline \multirow[t]{2}{*}{ Lead } & $\mathrm{L} \alpha$ & 10.549 & 10.37 to 11.32 \\
\hline & $\mathrm{L} \beta$ & 12.620 & 12.33 to 13.24 \\
\hline
\end{tabular}

${ }^{a}$ Copper $\mathrm{K} \alpha$, copper $\mathrm{K} \beta$ and tungsten $\mathrm{L} \alpha$ regions of interest overlap.

${ }^{b}$ Gold L $\alpha$ and platinum L $\alpha$ regions of interest overlap. 
Table 2. Conservative estimations of the upper limit of the minimum detection limit (MDL) of elements detected in the excitation/detection volume surrounding Particle B.

\begin{tabular}{lccccc}
\hline Element & Line & $I{\text { (counts })^{a}}$ & $\% \mathrm{C}_{\mathrm{i}}$ & $\begin{array}{c}\text { Calculated } \\
\text { Mass (ng) }\end{array}$ & MDL (ng) \\
\hline $\mathrm{Cr}$ & $\mathrm{K} \alpha$ & 89 & 32 & 1355 & 430 \\
$\mathrm{Fe}$ & $\mathrm{K} \alpha$ & 86437 & 3 & 1484 & 51.3 \\
$\mathrm{Ni}$ & $\mathrm{K} \alpha$ & 579 & 12 & 1679 & 209 \\
$\mathrm{Cu}$ & $\mathrm{K} \beta$ & 414 & 15 & 1689 & 249 \\
\hline
\end{tabular}

${ }^{a}$ Net intensity. 